\title{
Evaluation of Aromatic Boronic Acids as Ligands for Measuring Diabetes Markers on Carbon Nanotube Field-Effect Transistors
}

\author{
Steingrimur Stefansson, ${ }^{1}$ Lára A. Stefansson, ${ }^{2}$ Suk-won Chung, ${ }^{3}$ Kevin Ko, ${ }^{4}$ \\ Hena H. Kwon, ${ }^{5}$ and Saeyoung Nate Ahn ${ }^{1}$ \\ ${ }^{1}$ Fuzbien Technology Institute, 9700 Great Seneca Highway, Suite 302, Rockville, MD 20850, USA \\ ${ }^{2}$ Magruder High School, 5939 Muncaster Mill Road, Rockville, MD 20855, USA \\ ${ }^{3}$ Centreville High School, 6001 Union Mill Road, Clifton, VA 20124, USA \\ ${ }^{4}$ Thomas Jefferson High School for Science and Technology, 6560 Braddock Road, Alexandria, VA 22312, USA \\ ${ }^{5}$ MediSourcePlus Co., Ltd., 222-22 Gurodong, Gurogu, Seoul 336-708, Republic of Korea
}

Correspondence should be addressed to Steingrimur Stefansson, stennistef@fuzbien.com and

Saeyoung Nate Ahn, nate@fuzbien.com

Received 7 October 2011; Revised 14 December 2011; Accepted 14 December 2011

Academic Editor: Andrei Kolmakov

Copyright (C) 2012 Steingrimur Stefansson et al. This is an open access article distributed under the Creative Commons Attribution License, which permits unrestricted use, distribution, and reproduction in any medium, provided the original work is properly cited.

Biomolecular detections performed on carbon nanotube field-effect transistors (CNT-FETs) frequently use reactive pyrenes as an anchor to tether bioactive ligands to the hydrophobic nanotubes. In this paper, we explore the possibility of directly using bioactive aromatic compounds themselves as CNT-FET ligands. This would be an efficient way to functionalize CNT-FETs since many aromatic compounds bind avidly to nanotubes, and it would also ensure that ligand-binding molecules would be brought in close proximity to the nanotubes. Using a model system consisting of pyrene, phenanthrene, naphthalene, or phenyl boronic acids immobilized on CNT-FET wafers, we show that all are able to bind glycated human serum albumin (gHSA), which is an important diabetes marker. Pyrene boronic acid proved to bind CNTs with the greatest apparent affinity as measured by gHSA impedance. Interestingly, gHSA CNT-FET signal intensity, which is proportional to amount of protein bound, remained essentially unchanged for all the boronic acids tested.

\section{Introduction}

A variety of biomolecular sensors have been developed that circumvent the need for labeled detector molecules such as secondary antibodies. Label-free detection technologies can thus dramatically cut down on the time and cost of clinical assays by removing the need for labeling and imaging. And increased real-time and quantitative information on patient biomarkers is a critical step towards efficient personalized medicine.

Label-free detection of biomolecules based on their charge can be achieved through field-effect transistors (FETs), including carbon nanotube field-effect transistors (CNT-FET). Single-walled carbon nanotubes (SW-CNTs) are manufactured nanomaterials that have great potential for producing superior electronic instrumentation and detectors for biomedical applications $[1,2]$. SW-CNTs are essentially rolled-up graphite sheets that retain their excellent semiconductor properties in addition to their polyaromatic chemical nature [3-5]. Noncovalent attachment of ligands to CNTs is less likely to damage their structure, but the concentration of ligands bound to the nanotubes is a critical factor that influences signal strength. High densities of adsorbed ligands can lessen the signal through molecular crowding and low ligand densities decrease the signal by binding fewer targets [6]. Attaching ligands to CNT-FETs in such a way to generate a strong and reproducible signal of a captured protein is not a trivial matter. Some factors, not encountered in most conventional solid-phase immunodetection methods, also need to be taken into account. For example, a robust 
signal strength and consistency requires close proximity of a captured protein to the CNTs because the charge of the captured protein influences the current carried by the nanotubes [7]. Additionally, ion concentrations of many biological samples can reduce CNT-FET sensitivity and limit their potential applicability $[8,9]$.

Glycated hemoglobin (HbAlc) is a well-established marker for measuring the long-term effect of elevated blood glucose, but levels of glycated human serum albumin (gHSA) are a reliable intermediate glycemic indicator and is a more accurate indicator of glycation in a number of situations including patients with gestational diabetes $[14,15]$, diabetic hemodialysis patients $[16,17]$, and in HIV-infected patients undergoing aggressive antiretroviral therapy [18]. A rapid test for gHSA as a monthly indicator of glycation could bring about a substantial healthcare cost savings as well as an increase in patient compliance [11].

Hence, the ideal ligands to functionalize CNT-FETs would be small, hydrophobic molecules that directly bind the nanotubes and their targets with high affinity. In this study we evaluated if bioactive aromatic compounds can serve this dual purpose. To examine this possibility we use a model system consisting of aromatic boronic acids (Figure 1). Immobilized boronic acids are commonly used in diabetes research and clinical assays because they bind glucose modified (glycated) proteins, which are elevated in diabetes. Protein glycation is a spontaneous and nonenzymatic reaction between protein primary amino groups and some sugars and is the first step in a complex cascade of chemical reactions that can eventually lead to formation of highly crosslinked and nonfunctional proteins. These deleterious modifications occurring on extravascular proteins that are important in maintaining vascular tone can lead to impaired circulation, which is the underlying cause of many diabetic complications [10].

An early product of the glycation process is an $\mathrm{N}$ linked fructosamine adduct [11]. This fructosamine-protein adduct has a 1,2 cis-diol which can form covalent ester bonds with boronic acids (Figure 2). Immobilized aminophenyl boronic acid is commonly used as an affinity ligand for glycated proteins because of this stereo-specific bond formation [12]. Aromatic boronic acids are also being developed as fluorescent reporter groups to directly measure glycated proteins in patient samples [13].

We found that the aromatic boronic acids immobilized on our CNT-FET wafers bind gHSA as monitored by the increase in impedance. Their apparent affinity for the CNTs was in the increasing order of phenyl $<$ naphthalene $<$ anthracene $<$ pyrene. The optimal coating concentration for pyrene boronic acid was $2 \mathrm{mM}$, followed by anthracene, naphthalene, and phenyl boronic acids at $14 \mathrm{mM}, 41 \mathrm{mM}$, and $70 \mathrm{mM}$, respectively.

Nanosensors that are able to monitor glucose concentrations have been reported $[19,20]$ and developing a functional CNT-FET sensor that can also measure the glycation index could herald the advent of millimeter-scale diabetes monitoring systems that could be a part of a comprehensive sensor for personalized diagnosis and treatment for diabetes.

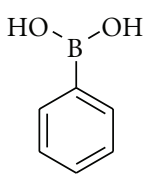

(a)

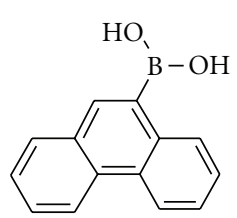

(c)

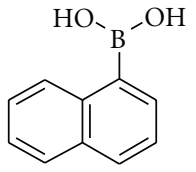

(b)

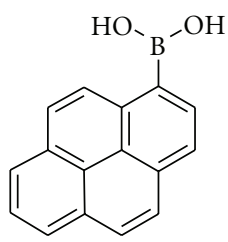

(d)
Figure 1: Structure of the boronic acids used in this study. (a) Phenyl boronic acid. (b) Naphthalene-1 boronic acid. (c) 9Phenanthrene boronic acid. (d) Pyrene-1 boronic acid.

\section{Materials and Methods}

2.1. Materials. Single-walled carbon nanotubes containing about $70 \%$ conducting nanotubes with diameters between 0.7 to $1.4 \mathrm{~nm}$ and length between 20 to $80 \mathrm{~nm}$ were purchased from Carbon Nanotechnologies Inc. 92 sample-well CNT wafers were manufactured by NanoPlatform Inc. using standard photolithography and lift-off process. 9-Phenanthrene boronic acid, naphthalene-1 boronic acid, sorbitol, dimethyl formamide, and ethanol were purchased from VWR. Pyrene boronic acid, phenyl boronic acid, glycated human serum albumin (contains $1-5$ mol fructosamine per mol albumin), and MOPS were purchased from Sigma-Aldrich. All chemicals were used as received.

\subsection{Measuring Glycated Human Serum Albumin Using Car-} bon Nanotube Field Effect Transistors. We have developed and manufactured an inexpensive CNT-FET wafer-based biosensor and have used it to measure serum insulin-like growth factor-1 (IGF-1) in a preclinical mouse model of human Brcal-related breast cancer. A description of the 92 circuit CNT-FET wafers used for this study and their characteristics were previously described by Jones et al. [21]. The aromatic boronic acids used in this study (Figure 1) were dissolved in DMF. Optimal coating concentrations of the boronic acids were determined by performing serial dilutions in DMF and applying them to the CNT-FET. $4 \mu \mathrm{L}$ of the DMF mixtures were incubated with CNT circuits for $30 \mathrm{~min}$ at RT in a closed container to prevent evaporation. After incubation, the wafers were briefly washed with ethanol to remove excess reagent and then air dried for $1 \mathrm{hr}$ at RT prior to use. For the assays, a baseline impedance value for the circuit was obtained after adding $4 \mu \mathrm{L}$ of $0.1 \mathrm{M}$ MOPS $\mathrm{pH} 7.5,5 \mathrm{mM} \mathrm{MgCl}_{2}$ (binding buffer) to the circuit for approximately $30 \mathrm{sec}$, after which $4 \mu \mathrm{L}$ of glycated human serum albumin (gHSA) in the same buffer was carefully admixed on the circuit and change in impedance measured for 2 additional minutes. The impedance value for each gHSA measurement was normalized to the corresponding buffer baseline value. Each sample was measured at least in 


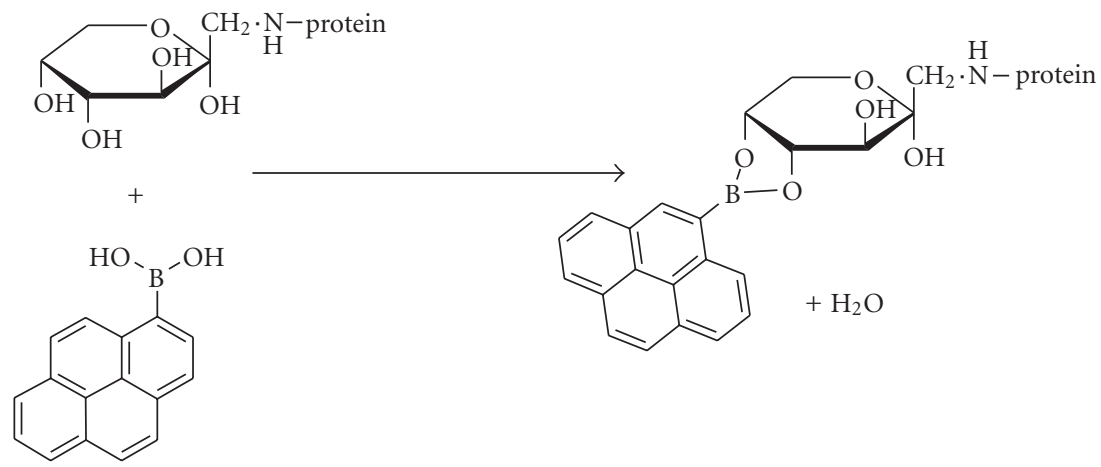

FIGURE 2: Formation of covalent ester bonds between pyrene boronic acid and a 1,2-cis diol. The cis-diol is depicted as a fructosamine-protein adduct. The boronic acid is planar and can therefore only form a bond with planar cis-diols.

quadruplicate using a fresh circuit for each measurement. A source/drain bias of $100 \mathrm{mV}$ was maintained throughout the measurements of the electrical signal and the pulse width was $1 \mathrm{sec}$. The reference electrode is the back (bottom) side of the grounded wafer. The device uniformity was not optimized for entire wafers, but individual circuits used for the assays were carefully evaluated before experiments. The selected CNT-FET circuits ranged typically between five and ten in on-off ratio. The electrical properties of the samples binding the CNT-FET were measured using a low-current measurement system (MediSourcePlus Inc.) that makes electrical contact to the source and drain electrodes of the CNTFET. The transfer characteristics of this circuit design were previously characterized for detection of prostate-specific antigen and IGF-1 $[6,21]$. Briefly, typical observed electronic transfer changes from 20 to 10 nanoamperes before and after the antibody immobilization on the CNT-FET circuits when $V_{\mathrm{ds}}$ and $V_{G}$ are 0.1 and -0.1 volt, respectively. The response in the electrical signal is typically in the range of 2 to $15 \%$ in the normalized units. A typical response of gHSA binding to the pyrene boronic acid immobilized on CNT-FET is shown in Figure 3. Unpaired $t$-test was performed using GraphPad online calculator.

\section{Results and Discussion}

Noncovalent functionalization of carbon nanotubes using 1-pyrenebutanoic acid succinimidyl ester has emerged as the method of choice for ATTACHING BIOLOGICAL molecules such as antibodies and aptamers to CNT-FETs [22-24]. In practice, the CNTs are allowed to absorb the hydrophobic pyrene followed by removal of excess unbound pyrene and addition of protein which reacts with the succinimidyl ester moiety [25]. Using reactive pyrenes for immobilizing biological molecules onto CNTs is a simple and convenient procedure, and they are readily available from many commercial vendors. However, these reactive pyrene compounds were initially developed as labels for fluorescent polarization studies due to the long lifetime of the excited state of pyrene [26, 27]. Pyrene butanoic acid succinimidyl ester has a 4-carbon spacer arm separating the amine reactive succinimidyl ester from the pyrene moiety. Incorporating spacers on fluorescent protein labels is a practical matter

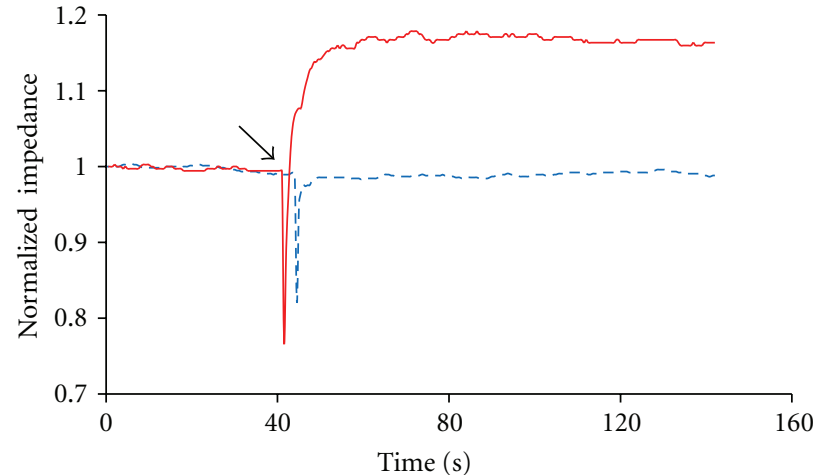

FIGURE 3: Representative real-time change in impedance upon binding of $5 \mu \mathrm{g} / \mathrm{mL}$ gHSA to pyrene-1 boronic acid coated CNTFET circuit (solid red line) and to noncoated CNT-FET circuit (dotted blue line). Arrow indicates the time of sample application.

since they can increase access of bulky and hydrophobic fluorescent groups to protein surface groups while minimizing any direct effect labels have on protein structure and vice versa. Using a spacer for CNT-FET detection has potential drawbacks because the interactions of the ligand and target occur further from the nanotube surface and will diminish the signal [8]. This issue is acerbated by the high salt concentration in biological samples because ions shield the charge effect that captured proteins have on the CNT current (Debye length). The Debye length is defined as the distance after which mobile charges, such as ions, will screen out the electric field strength. It is inversely proportional to the square root of the ionic concentration of the medium so at physiological ion concentrations $(\sim 150 \mathrm{mM})$ the Debye length will be around 1 nanometer, which is close to the length of a 4-carbon spacer [28, 29]. In some cases reducing the salt concentration can resolve the issue of distance [30, 31]. In other cases this would not be an option because reducing the ionic strength of biological samples can cause protein denaturation and/or precipitation in addition to cell lysis if whole blood is being assayed. Therefore using bioactive aromatic compounds directly as CNT-FET ligands could offer a simple solution to these issues. 


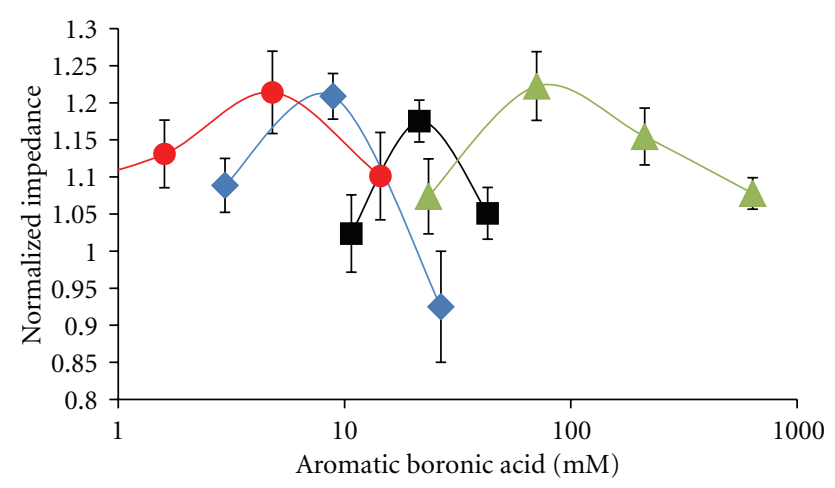

FIGURE 4: Representative partial titration curves showing the peak coating concentrations of aromatic boronic acids on CNTFET circuits. Detection of bound boronic acids was achieved by measuring the CNT-FET electrical properties in the presence of $5 \mu \mathrm{g} / \mathrm{mL}$ gHSA. From left to right, the curves are pyrene- 1 boronic acid (red ), 9-phenanthrene boronic acid (blue $\$$ ), naphthalene-1 boronic acid (black $\boldsymbol{\square}$ ), phenyl boronic acid (green $\boldsymbol{\Delta}$ ). Each partial titration curve shows at least 3 concentrations of the boronic acids with each point measured in quadruplicate \pm standard error of the mean.

To evaluate the utility of aromatic boronic acids as direct CNT-FET ligands, we first determined the optimal coating concentrations for the boronic acids. Stock solutions of the aromatic boronic acids were made in dimethyl formamide (DMF) and titrated 3-fold in DMF down to approximately $0.05 \mathrm{mM}$ after which aliquots of the dilutions were added to CNT-FET wafers as described in Section 2. After incubation and a brief wash with ethanol followed by drying, the binding of gHSA was measured as described in the Section 2. The concentration of gHSA used in this study $(5 \mu \mathrm{g} / \mathrm{mL})$ is well within the range of plasma gHSA in normoglycemia and hyperglycemia, which are approximately $14 \%$ and $25 \%$ of total serum albumin (4-5 g/liter), respectively [32]. Furthermore, changing the ionic strength of the buffer used for these studies did not significantly influence the CNT-FET signal upon gHSA binding to pyrene boronic acid coated circuits, which indicates that the gHSA is in close proximity to the CNT surface (data not shown).

Figure 3 shows typical real-time binding of gHSA to CNT-FET wafers coated with $2 \mathrm{mM}$ pyrene boronic acid compared to control, which is gHSA added to uncoated CNT-FET. Higher than optimal coating concentrations of the boronic acids resulted in lower impedance of gHSA, possibly due to aggregation of the compounds onto the CNTs during the ethanol wash. Furthermore, extensive washing of the CNT-FET wafers with ethanol spray removed the smaller phenyl and naphthalene aromatic boronic acids from the wafers (data not shown). In order to perform the experiments with all aromatic boronic acids, the washing step consisted of rapidly immersing the wafers into a $95 \%$ ethanol bath and air drying.

Figure 4 shows representative partial titration curves of each of the aromatic boronic acids onto CNT-FETs followed by addition of $5 \mu \mathrm{g} / \mathrm{mL}$ gHSA and demonstrates an increase in impedance upon binding of the protein.
TABle 1: Summary of the binding studies performed with the aromatic boronic acids on the CNT-FET wafers. The studies were performed as described in the text and represent the average of at least 2 titration curves for each boronic acid. Each titration curve represents at least 5 concentration points of the boronic acids with each point measured in quadruplicate.

\begin{tabular}{lcc}
\hline Boronic acids & $\begin{array}{c}\text { Optimal coating } \\
\text { concentration }\end{array}$ & $\begin{array}{l}\text { Peak impedance } \\
\text { value of gHSA }\end{array}$ \\
\hline $\begin{array}{l}\text { Phenyl boronic acid } \\
\text { Naphthalene boronic }\end{array}$ & $70 \mathrm{mM}( \pm 6.2 \mathrm{mM})$ & $12.5 \%( \pm 5.4 \%)$ \\
$\begin{array}{l}\text { acid } \\
\text { Phenanthrene boronic } \\
\text { acid }\end{array}$ & $14 \mathrm{mM}( \pm 7.8 \mathrm{mM})$ & $10.6 \%( \pm 3.2 \%)$ \\
Pyrene boronic acid & $2 \mathrm{mM}( \pm 1.2 \mathrm{mM})$ & $12.2 \%( \pm 2.8 \%)$ \\
\hline
\end{tabular}

* Impedance increases were assessed at the optimal coating concentrations for each boronic acid and represent the percent increase in the impedance compared to baseline.

The increase in impedance upon binding of gHSA to boronic acid coated CNT-FET circuits is likely due to the interactions of electron donating primary amine groups of proteins with the CNTs, which has been shown to decrease conductance (e.g., increase impedance) [33-36]. Table 1 summarizes the results of these experiments for all the aromatic boronic acids and demonstrates that the observed optimal coating concentrations were lowest for pyrene-1 boronic acid ( $2 \mathrm{mM})$, followed by $14 \mathrm{mM}$ for 9phenanthracenyl boronic acid, $41 \mathrm{mM}$ for naphthalene-1 boronic acid and $70 \mathrm{mM}$ for phenyl boronic acid. These values are in good agreement with other observations, including those of Yoo et al. [37] that demonstrated that retention of aromatics on immobilized single-walled CNTs solid-phase column, which is a qualitative metric for binding affinities was benzene < naphthalene $<$ phenanthrene $<$ pyrene.

To verify the specificity of the assay, CNT-FETs wafers were coated with pyrene boronic acid at optimal coating concentration, and the impedance was measured with different gHSA concentrations. Figure 5(a) shows the response of the CNT-FET and demonstrates a concentration-dependant increase in the gHSA signal. Further verification of the specificity of the assay was performed by measuring gHSA binding on CNT-FETs coated with the aromatic boronic acids in the presence of $20 \mathrm{mM}$ sorbitol (Figure 5(b)). Sorbitol is a 6-carbon polyalcohol that contains a cis-diol and is frequently used as a competitive ligand to elute glycated proteins from boronic acid affinity columns [38]. As seen in Figure 5(b), inclusion of excess sorbitol in the assay inhibits the binding of gHSA to the boronic acid coated CNT-FET wafers.

Interestingly, there was little difference in the gHSA signal amplitude, with Table 1 showing that the impedance was elevated between $10 \%$ and $15 \%$ regardless of which aromatic boronic acid was coated onto the CNT-FET wafers. One possibility for these results is that the aromatic boronic acids used in this study are planar and rigid AND will likely form $\pi-\pi$ interactions with CNT sidewalls which offer the least 


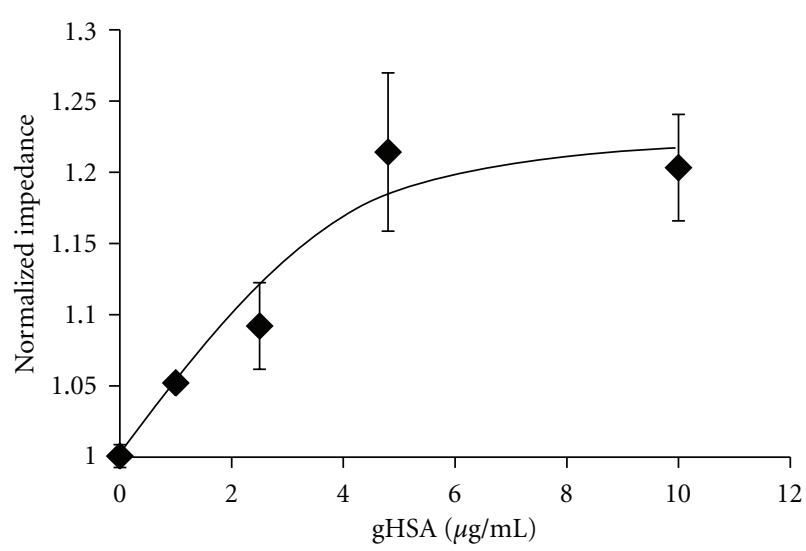

(a)

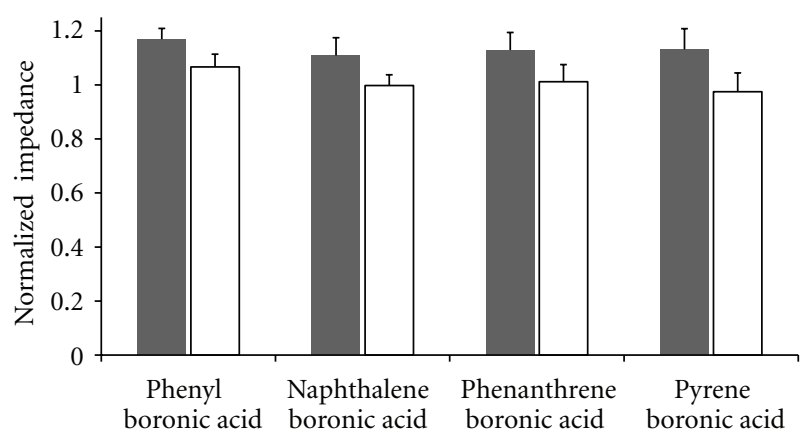

(b)

Figure 5: Titration of gHSA onto CNT-FET circuits coated with $2 \mathrm{mM}$ pyrene boronic acid. (a) Inhibition of gHSA binding to aromatic boronic acids coated onto CNTs (dark bars) by addition of sorbitol (white bars). (b) $0.1 \mathrm{M}$ MOPS buffer containing $20 \mathrm{mM}$ sorbitol was incubated on the circuits for 30 seconds before addition of $10 \mu \mathrm{g} / \mathrm{mL}$ gHSA containing $20 \mathrm{mM}$ sorbitol, as described in Section 2. Each sample was measured in at least hexuplicate. Unpaired $t$-test showed significance $P<0.05$ for all sorbitol additions.

curvature and thus the largest surface area available for $\pi$ $\pi$ interactions. A similar observation was inferred from the interactions of the cancer drug doxorubicin with singlewall CNTs of different diameters [39]. Doxorubicin, like pyrene boronic acid, is a planar polycyclic and hydrophobic compound that adheres readily to CNTs. However, the release of doxorubicin into solution from CNT preparations with an average diameter of $1.3 \mathrm{~nm}$ was faster than from CNTs with an average diameter of $1.9 \mathrm{~nm}$. This difference was surmised to be due to a more favorable $\pi$-stacking of doxorubicin onto larger diameter CNTs because they have flatter sidewalls. In comparison, doxorubicin is retained much more readily on graphene because of its flat structure [40].

\section{Conclusion}

It is our opinion that ideal CNT-FET ligands for detecting and measuring biological targets should be small and hydrophobic. These attributes would allow for efficient CNT coating and assure that the target is binding the ligand in close proximity to the CNT surface. Additionally, the close binding would not require dilution of the biological sample to reduce ion concentrations. In the present study we demonstrate that aromatic boronic acids can be used directly as CNT-FET ligands for detecting diabetes markers. Our data indicates that of these compounds, pyrene boronic acid was superior to the smaller aromatics with respect to retention, but the performance of all aromatic boronic acids were similar in their ability to bind gHSA and were not adversely affected by the buffer concentration in the assay. Further studies are required to assess the performance of the CNT-FET wafers for measuring the concentrations of glycated protein markers in a clinical setting.

\section{Acknowledgments}

This paper was supported in part by the Maryland Industrial Partnership (MIPS) program, no. 4319 and by the Maryland Technology Development Corporation's Maryland Technology Transfer and Commercialization Fund.

\section{References}

[1] P. Vichchulada, Q. Zhang, and M. D. Lay, "Recent progress in chemical detection with single-walled carbon nanotube networks," Analyst, vol. 132, no. 8, pp. 719-723, 2007.

[2] D. R. Kauffman and A. Star, "Electronically monitoring biological interactions with carbon nanotube field-effect transistors," Chemical Society Reviews, vol. 37, no. 6, pp. 11971206, 2008.

[3] C. Soldano, A. Mahmood, and E. Dujardin, "Production, properties and potential of graphene," Carbon, vol. 48, no. 8, pp. 2127-2150, 2010.

[4] P. Avouris, "Molecular electronics with carbon nanotubes," Accounts of Chemical Research, vol. 35, no. 12, pp. 1026-1034, 2002.

[5] D. A. Britz and A. N. Khlobystov, "Noncovalent interactions of molecules with single walled carbon nanotubes," Chemical Society Reviews, vol. 35, no. 7, pp. 637-659, 2006.

[6] J. P. Kim, B. Y. Lee, J. Lee, S. Hong, and S. J. Sim, "Enhancement of sensitivity and specificity by surface modification of carbon nanotubes in diagnosis of prostate cancer based on carbon nanotube field effect transistors," Biosensors and Bioelectronics, vol. 24, no. 11, pp. 3372-3378, 2009.

[7] J. P. Kim, B. Y. Lee, S. Hong, and S. J. Sim, "Ultrasensitive carbon nanotube-based biosensors using antibody-binding fragments," Analytical Biochemistry, vol. 381, no. 2, pp. 193$198,2008$.

[8] M. R. Leyden, C. Schuman, T. Sharf, J. Kevek, V. T. Remcho, and E. D. Minot, "Fabrication and characterization of carbon nanotube field-effect transistor biosensors," in Proceedings of the Organic Semiconductors in Sensors and Bioelectronics III, R. Shinar and I. Kymissis, Eds., vol. 7779, San Diego, Calif, USA, August 2010.

[9] K. Balasubramanian, E. J. H. Lee, R. T. Weitz, M. Burghard, and K. Kern, "Carbon nanotube transistors-chemical functionalization and device characterization," Physica Status Solidi A, vol. 205, no. 3, pp. 633-646, 2008.

[10] S. Y. Goh and M. E. Cooper, "The role of advanced glycation end products in progression and complications of diabetes," Journal of Clinical Endocrinology and Metabolism, vol. 93, no. 4, pp. 1143-1152, 2008. 
[11] H. V. Roohk and A. R. Zaid, "A review of glycated albumin as an intermediate glycation index for controlling diabetes," Journal of Diabetes Science and Technology, vol. 2, no. 6, pp. 1114-1121, 2008.

[12] K. Mallia, G. T. Hermanson, R. I. Krohn, E. K. Fujimoto, and P. K. Smith, "Preparation and use of a boronic acid affinity support for separation and quantitation of glycosylated hemoglobins," Analytical Letters, vol. 14, no. 8, pp. 649-661, 1981.

[13] G. Kaur, N. Lin, H. Fang, and B. Wang, "Boronic acidbased fluorescence sensors for glucose monitoring," Topics in Fluorescence Spectroscopy, vol. 11, pp. 377-397, 2006.

[14] F. Abe, N. Miyamoto, Y. Tahara, J. Takahashi, and K. Shima, "Serum glycated albumin concentrations during pregnancy," Annals of Clinical Biochemistry, vol. 30, no. 2, pp. 198-200, 1993.

[15] J. M. Hicks, R. Haeckel, C. P. Price, K. Lewandrowski, and A. $\mathrm{H}$. Wu, "Recommendations and opinions for the use of pointof-care testing for hospitals and primary care: summary of a 1999 symposium," Clinica Chimica Acta, vol. 303, no. 1-2, pp. 1-17, 2001.

[16] M. Inaba, S. Okuno, Y. Kumeda et al., "Glycated albumin is a better glycemic indicator than glycated hemoglobin values in hemodialysis patients with diabetes: effect of anemia and erythropoietin injection," Journal of the American Society of Nephrology, vol. 18, no. 3, pp. 896-903, 2007.

[17] T. P. Peacock, Z. K. Shihabi, A. J. Bleyer et al., "Comparison of glycated albumin and hemoglobin $\mathrm{A}_{1 \mathrm{c}}$ levels in diabetic subjects on hemodialysis," Kidney International, vol. 73, no. 9, pp. 1062-1068, 2008.

[18] P. S. Kim, C. Woods, P. Georgoff et al., " $A_{1 C}$ underestimates glycemia in HIV infection,” Diabetes Care, vol. 32, no. 9, pp. 1591-1593, 2009.

[19] C. B. Jacobs, M. J. Peairs, and B. J. Venton, "Review: carbon nanotube based electrochemical sensors for biomolecules," Analytica Chimica Acta, vol. 662, no. 2, pp. 105-127, 2010.

[20] K. Besteman, J.-O. Lee, F. G. M. Wiertz, H. A. Heering, and C. Dekker, "Enzyme-coated carbon nanotubes as singlemolecule biosensors," Nano Letters, vol. 3, no. 6, pp. 727-730, 2003.

[21] L. P. Jones, S. Stefansson, M. Kim, and S. N. Ahn, "Comparison of radioimmuno and carbon nanotube field-effect transistor assays for measuring insulin-like growth factor-1 in a preclinical model of human breast cancer," Journal of Nanobiotechnology, vol. 9. article 36, 2011.

[22] A. Star, J. C. P. Gabriel, K. Bradley, and G. Grüner, "Electronic detection of specific protein binding using nanotube FET devices," Nano Letters, vol. 3, no. 4, pp. 459-463, 2003.

[23] K. Maehashi, T. Katsura, K. Kerman, Y. Takamura, K. Matsumoto, and E. Tamiya, "Label-free protein biosensor based on aptamer-modified carbon nanotube field-effect transistors," Analytical Chemistry, vol. 79, no. 2, pp. 782-787, 2007.

[24] P. A. Hu, J. Zhang, L. Li, Z. Wang, W. O’Neill, and P. Estrela, "Carbon nanostructure-based field-effect transistors for labelfree chemical/biological sensors," Sensors, vol. 10, no. 5, pp. 5133-5159, 2010.

[25] R. J. Chen, Y. Zhang, D. Wang, and H. Dai, "Noncovalent sidewall functionalization of single-walled carbon nanotubes for protein immobilization," Journal of the American Chemical Society, vol. 123, no. 16, pp. 3838-3839, 2001.

[26] J. K. Weltman, R. P. Szaro, A. R. Frackelton Jr., R. M. Dowben, J. R. Bunting, and B. E. Cathou, "N-(3-pyrene)maleimide: a long lifetime fluorescent sulfhydryl reagent," Journal of Biological Chemistry, vol. 248, no. 9, pp. 3173-3177, 1973.
[27] J. F. Eccleston, J. P. Hutchinson, and D. M. Jameson, "Fluorescence-based assays," Progress in Medicinal Chemistry, vol. 43, pp. 19-48, 2005.

[28] F. Tessier and G. W. Slater, "Effective Debye length in closed nanoscopic systems: a competition between two length scales," Electrophoresis, vol. 27, no. 3, pp. 686-693, 2006.

[29] C. Heitzinger and G. Klimeck, "Computational aspects of the three-dimensional feature-scale simulation of siliconnanowire field-effect sensors for DNA detection," Journal of Computational Electronics, vol. 6, no. 1-3, pp. 387-390, 2007.

[30] E. Stern, R. Wagner, F. J. Sigworth, R. Breaker, T. M. Fahmy, and M. A. Reed, "Importance of the Debye screening length on nanowire field effect transistor sensors," Nano Letters, vol. 7, no. 11, pp. 3405-3409, 2007.

[31] G. Zheng, F. Patolsky, Y. Cui, W. U. Wang, and C. M. Lieber, "Multiplexed electrical detection of cancer markers with nanowire sensor arrays," Nature Biotechnology, vol. 23, no. 10, pp. 1294-1301, 2005.

[32] P. Rondeau and E. Bourdon, "The glycation of albumin: structural and functional impacts," Biochimie, vol. 93, no. 4, pp. 645-658, 2011.

[33] K. Bradley, M. Briman, A. Star, and G. Gruner, "Charge transfer from adsorbed proteins," Nano Letters, vol. 4, no. 2, pp. 253-256, 2004.

[34] K. Bradley, J. C. P. Gabriel, M. Briman, A. Star, and G. Grüner, "Charge transfer from ammonia physisorbed on nanotubes," Physical Review Letters, vol. 91, no. 21, pp. 218301-218304, 2003.

[35] H. Chang, J. D. Lee, S. M. Lee, and Y. H. Lee, "Adsorption of $\mathrm{NH}_{3}$ and $\mathrm{NO}_{2}$ molecules on carbon nanotubes," Applied Physics Letters, vol. 79, no. 23, pp. 3863-3865, 2001.

[36] K. Teker, E. Wickstrom, and B. Panchapakesan, "Biomolecular tuning of electronic transport properties of carbon nanotubes via antibody functionalization," IEEE Sensors Journal, vol. 6, no. 6, pp. 1422-1427, 2006.

[37] J. T. Yoo, H. Ozawa, T. Fujigaya, and N. Nakashima, "Evaluation of affinity of molecules for carbon nanotubes," Nanoscale, vol. 3, no. 6, pp. 2517-2522, 2011.

[38] N. Iberg and R. Fluckiger, "Nonenzymatic glycosylation of albumin in vivo. Identification of multiple glycosylated sites," Journal of Biological Chemistry, vol. 261, no. 29, pp. 1354213545, 1986.

[39] Z. Liu, X. Sun, N. Nakayama-Ratchford, and H. Dai, "Supramolecular chemistry on water-soluble carbon nanotubes for drug loading and delivery," ACS Nano, vol. 1, no. 1, pp. 50-56, 2007.

[40] X. Yang, X. Zhang, Z. Liu, Y. Ma, Y. Huang, and Y. Chen, "High-efficiency loading and controlled release of doxorubicin hydrochloride on graphene oxide," Journal of Physical Chemistry C, vol. 112, no. 45, pp. 17554-17558, 2008. 

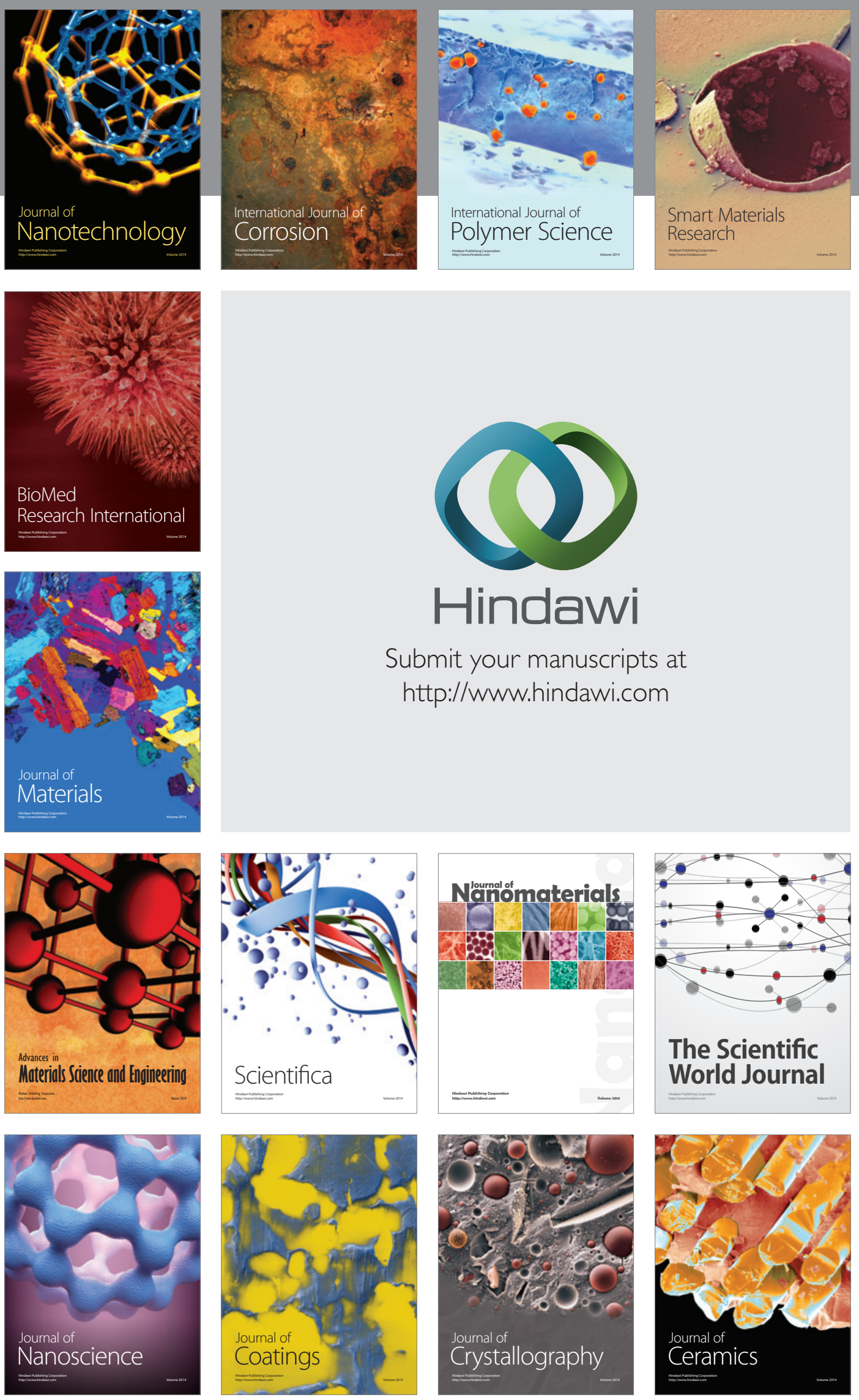

The Scientific World Journal

Submit your manuscripts at

http://www.hindawi.com

\section{World Journal}

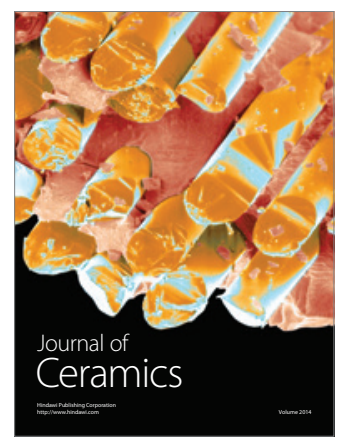

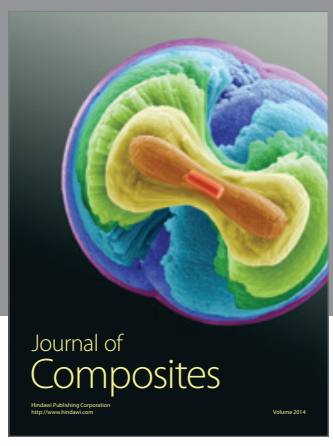
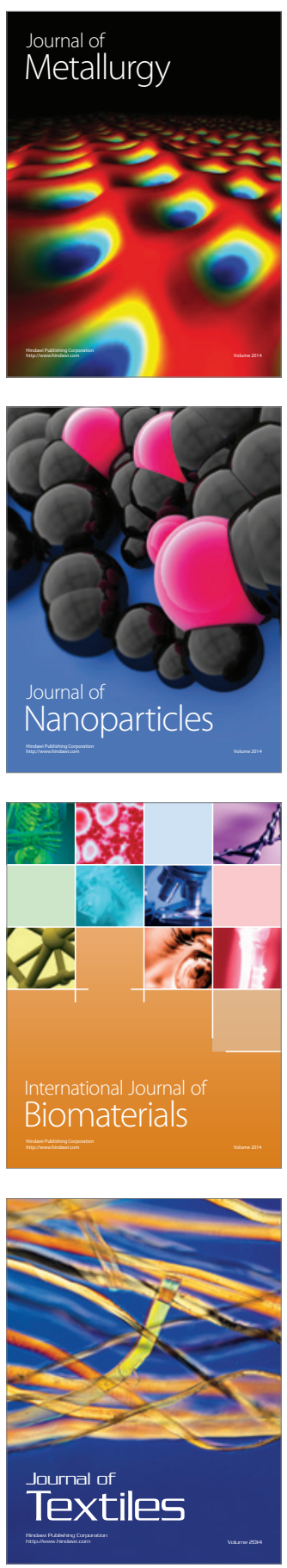